\title{
Ocorrência de Salmonella spp. em carcaças de frangos recém abatidos em feiras e mercados da cidade de Manaus-AM
}

\author{
Izabel Cristina Campos TIROLLI ${ }^{1}$, Cristóvão Alves da COSTA²
}

\begin{abstract}
RESUMO
A presente pesquisa avaliou a contaminação em carcaças de frango por salmonelas. O estudo foi realizado em feiras e mercados nas diferentes zonas da Cidade de Manaus-AM, por um período de 11 semanas (março a maio de 1998). Sessenta amostras foram examinadas; destas, trinta (50\%) resultaram positivas para Salmonella spp., das quais foram isoladas 67 cepas, incluídas em 11 sorotipos diversos. Entre os sorotipos identificados, a S. panama, S. mbandaka, S. schwarzengrund, S. typhimurium, S. albany e $S$. agona, foram as predominantes, representando $85 \%$ do total isolado. A contaminação de Salmonella é dependente de vários fatores, e a sua ocorrência pode estar relacionada com as condiçóes de higiene da granja. A metodologia empregada para a deteç̧ão microbiológica foi a recomendada pela "Food and Drug Administration”, dos Estados Unidos da América.
\end{abstract}

\section{PALAVRAS-CHAVE}

Salmonella spp, carcaças de frango, aves, microbiologia.

\section{Occurrence of Salmonella spp. in chicken carcasses commercialized in open markets in the city of Manaus - Amazonas}

\section{ABSTRACT}

This research evaluates the levels of salmonella that are present in chicken carcasses commercialized in shops and public markets in different zones in the city of Manaus, Amazonas - Brazil. Sixty samples were randomly collected in a period of eleven weeks and examined specifically for Salmonella presence. Fifty percent of the samples were positive for Salmonella, and 67 serovars were isolated and distributed mainly among 11 different sorotypes. Those identified, S. panama, S. mbandaka, S. schwarzengrund, S. typhimurium, S. albany, S. agona, corresponded to $85 \%$. The contamination of Salmonella is dependent on various factors, and its occurrence may berelated to the hygeine conditions of the poultry farm. The methodology used for the microbiological detection was that recommende by the "Food and Drugs Administration" of the United States of America.

\section{KEY WORDS}

Salmonella spp, carcasses chicken, poultry, microbiology.

\footnotetext{
${ }^{1}$ Universidade Federal do Amazonas, Departamento de Ciências de Alimentos - Rua Alexandre Amorim, 330 - Aparecida -CEP: 69010-300 - Manaus/AM. 


\section{INTRODUÇÃO}

As salmonelas são as maiores responsáveis por toxinfecções alimentares humana. No mundo elas representam cerca de 10$15 \%$ de casos de gastroenterite aguda. São fontes mais comuns destes surtos alimentares, as aves, ovos, carnes, produtos de carne e chocolate (Jay, 2000).

No Brasil, são escassos os dados epidemiológicos mostrando a importância do problema das salmoneloses em humanos, uma vez que a notificação de intoxicação por alimentos contaminados não é obrigatória (Piccollo et al. 1992). No entanto, levantamentos efetuados nos Estados Unidos estimam entre 2 a 4 milhões o número de casos anuais desta doença. De acordo com o "United States Department of Human Service Center for Disease Control", em Atlanta, 1/3 dos casos de doenças transmitidas por alimentos é devido a Salmonella spp (Food and Drug administration - FDA, 2003). Além do problema no âmbito da saúde pública, a salmonelose provoca danos consideráveis na área econômica. Cerca de 4 bilhōes de dólares é o prejuízo anual à economia norte americana decorrente do problema da salmonelose no homem e em animais (Mead et al. 1999).

Intoxicações alimentares causadas por Salmonella spp ocorrem mesmo em países desenvolvidos. No Brasil, supõe-se que a ocorrência de salmonelas seja relevante devido as deficiências de saneamento básico e as más condiçôes higiênico-sanitárias da maioria da população, aliadas ao precário controle de qualidade de algumas indústrias alimentícias e de pequenos abatedouros de aves (Fuzihara et al. 2000).

Como a região Amazônica está distante dos grandes centros industriais, o problema persiste com maior gravidade, devido ao processamento de alguns alimentos ser puramente caseiro e empírico. Considerando-se que existem diversos pontos comerciais que abatem e comercializam aves em condiçôes higiênicas sanitárias insatisfatórias, objetivou-se nesse trabalho avaliar o nível de contaminação de Salmonella spp em carcaças de frango abatidas e comercializadas em feiras e mercados da Cidade de Manaus-AM.

\section{MATERIAL E MÉTODOS}

\section{OBTENÇÃO DAS AMOSTRAS}

As carcaças de frangos foram coletadas em feiras livres e mercados registrados na prefeitura municipal de Manaus situados nas diferentes zonas da cidade (Norte, Sul, Leste, Oeste, CentroSul e Centro-Oeste), no período de março a maio de 1998. Foram colhidas dez amostras de cada zona. Os frangos analisados eram abatidos, limpos, eviscerados e embalados no local da venda. As amostras coletadas eram identificadas e acondicionadas em embalagens estéreis e transportadas ao laboratório para análise imediata.

\section{PROCESSAMENTO DAS AMOSTRAS}

As amostras foram retiradas assepticamente das embalagens e transferidas para sacos plásticos estéreis contendo $500 \mathrm{ml} \mathrm{de} \mathrm{caldo}$ lactosado, enxaguadas vigorosamente por cerca de 1 minuto e mantidas na água de enxágüe por mais 30 minutos. Utilizou-se a técnica de enxágüe ( $\mathrm{D}^{\prime}$ Aoust et al., 1982)

\section{PESQUISA DE Salmonella SP}

As amostras foram pré-enriquecidas com a adição de $225 \mathrm{ml}$ de caldo lactosado e incubadas à $35^{\circ} \mathrm{C}$ por 24 horas. Após a incubação foi feito enriquecimento seletivo, transferindo-se respectivamente $0,1 \mathrm{ml} \mathrm{e} 1 \mathrm{ml}$ da cultura pré-enriquecida para dois tubos, um contendo $10 \mathrm{ml}$ de Caldo Rappaport-Vassiliadis (RV) e outro com $10 \mathrm{ml}$ de caldo Tetrationato verde brilhante (TBG) suplementado com $0,1 \mathrm{ml}$ de solução de verde brilhante 1:1000 e $0,2 \mathrm{ml}$ de solução de iodo iodeto. Os tubos foram incubados à $35^{\circ} \mathrm{C} \mathrm{e} 42^{\circ} \mathrm{C}$ por 24 horas, respectivamente.

Os caldos de enriquecimento seletivo foram semeados em duas placas, uma contendo ágar Hektoen Enteric (HE) e outra ágar Sulfito de bismuto (BSA). Incubaram-se as placas à $35^{\circ} \mathrm{C}$ por 24 horas. Foram selecionadas 2 (duas) colônias típicas para Salmonella de cada meio de cultura as quais foram isoladas em ágar triptona de soja (TSA) por $24 \mathrm{~h}$ a $35^{\circ} \mathrm{C}$, para serem submetidas à triagem bioquímica. (Andrews \& Hammack, 2003). As culturas com comportamento bioquímico típico, eram confirmadas com anti-soro somático polivalente. Posteriormente, para identificação dos sorotipos, eram confirmadas com antisoros específicos.

Os testes bioquímicos foram feitos através da metodologia tradicional (Andrews \& Hammack 2003) e metodologia automatizada. No método automatizado foram utilizadas placas de microtitulação com 21 reações bioquímicas, incubadas por $18 \mathrm{~h}$ a $24 \mathrm{~h} \mathrm{à} 35^{\circ} \mathrm{C}-37^{\circ} \mathrm{C}$. Após a incubação foram interpretadas por um sistema computadorizado e leitor óptico, indicando o microorganismo isolado.

\section{RESULTADOS E DISCUSSÃO}

Das 60 amostras de carcaças de frangos analisadas, 30 (50\%) foram positivas para Salmonella spp. Nessas condiçōes as amostras são consideradas impróprias para consumo humano (Jay, 2000).

As taxas de contaminação em carcaças de frango são variáveis. Estudos realizados por diferentes pesquisadores demonstram resultados superiores aos encontrados no presente trabalho. Pesquisas realizadas na Espanha por Capita et al. (2003) demonstraram taxas de positividade de 55\%; e no Brasil por Almeida et al. (2000) positividade de $86,7 \%$. Alguns autores explicam que esta variação nos índices está relacionada com a procedência do lote (contaminação primária); condições higiênicosanitária dos abatedouros (área física e manipuladores), contaminação cruzada ocorrida nas áreas de depenagem, lavagem, 
resfriamento e embalagem. Após o processamento, durante a etapa de transporte e comercialização, as carcaças ainda estão sujeitas à contaminação adicional. (Corry et al. 2002; Mikolajczyk \& Radkowski, 2002; Olsen et al. 2003)

Das amostras positivas foram isoladas 480 cepas, sendo que apenas 67 (sessenta e sete) confirmaram-se sorologicamente positivas para Salmonella spp. Dentre as cepas isoladas foram identificados 11 sorotipos, sendo a mais freqüente a $S$. panama, com 22,4\%, seguidas de S. mbandaka, com 17,9\%; $S$. schwarzengrund, com 14,9\%; S. typhimurium, com 13,4\%; S. albany, com 10,4\%; S. agona, com 6\%; S. saintpaul, com 4,5\%; S. senftenberg, com 3\%; S. muechen, com 3\%; S. emek, com 3\% e S. ohio, com $1,5 \%$. Estas espécies estão enquadradas no grupo de salmonelas que causam enterocolites.

A alta incidência de Salmonella em aves vivas como também a contaminação cruzada durante o seu processamento, resultam na incriminação do frango como um dos principais veículos envolvidos em casos de salmoneloses humanas (Mulder, 1999; Bauemier et al. 2000; Daniels et al. 2002). Em estudo realizado na Bélgica Uyttendaele et al. (1999), analisando 772 carcaças de frango, constataram a contaminação de Salmonella spp em 36,5\% das amostras. Na Polônia, Mikolajczyk \& Radkowski, (2002), ao estudarem 4 diferentes etapas de processamento de carcaças de frango encontraram uma porcentagem alta de resultados positivos para Salmonella spp, de 400 amostras observaram 23,7\% de positividade, distribuídos nos processos de abate, pós evisceração, pré e pós resfriamento, sendo os tipos isolados $S$. enteritidis, S. typhimurium, S. saintpaul, S. agona e S. infantis, sendo a $S$. enteritidis o sorotipo mais dominante.

A S. typhimurium é o sorotipo de origem alimentar que se encontra com maior frequência em todo o mundo (Jay, 2000; Olsen et al. 2001; Mukesh \& Mukesh, 2002). Nesta pesquisa ocorreram em 13,4\% dos isolamentos. Em recentes estudos (Olsen et al. 2003), investigando a contaminação por Salmonella em um abatedouro avícola encontraram Salmonella typhimurium como o sorotipo mais freqüente.

A Salmonella enteritidis é o segundo sorotipo mais comumente encontrado em alimentos. Nos últimos anos, tem-se observado um aumento da incidência de salmonelose causada por S. enteritidis, envolvendo ovos e produtos a base de ovos. Esse sorotipo tem a peculiaridade de colonizar o canal ovopositor das galinhas, causando a contaminação da gema durante a formação do ovo (Landgraft \& Franco, 1996). É hoje em alguns países o principal sorotipo isolado de produtos avícolas e amostras clínicas de pacientes com gastroenterites devido a surtos alimentares (Capita et al. 2000; Olsen et al. 2001; dos Santos et al. 2003). Nas últimas duas décadas este sorotipo representou de $0,4-1 \%$ de todos os sorotipos isolados de infecção humana, mas a partir de 1993, passou a ser isolado com maior freqüência, alcançando em 1996, 55\% das cepas de materiais humanos e
32,7\% daqueles de origem não humana (Olsen et al. 2001; Tavechio et al. 2002). Em nossa pesquisa não foi detectado esse sorotipo.

Alguns pesquisadores relacionaram os sorotipos isolados em carcaças de frango com os sorotipos predominantemente isolados nas últimas décadas. Tavechio et al. (2002), observaram pelos resultados a semelhança entre os sorotipos predominantes nas carcaças (S. enteritidis-32,7\%, S. senftenberg-10,3\%, S. hadar -6,8\% e S. agona-5,1\% e, S. Typhimurium-2,4\%).

Duitschaever (1977) e Tibaijuka et al. (2003) analisando pedaços fatiados de frango encontraram respectivamente $S$. muechen $(34,8 \%)$ e $S$. saintpaul (14,8\%), entre os sorotipos mais freqüentes. Em nossa pesquisa tais isolamentos foram encontrados nas seguintes proporções: $S$. muechen (3\%) e S. saintpaul (4,5\%).

Nos mercados onde foram coletadas coletadas as amostras, a maior freqüência para Salmonella spp, em carcaças de frango recém-abatidos, ocorreu em amostras oriundas da Zona Sul (30\%), seguida da Centro-Oeste (23,3\%), Leste (16,7\%), Centro-Sul (13,3\%), Norte (10\%) e Oeste (6,7\%).

A contaminação de Salmonella sppé dependente de vários fatores, e a sua ocorrência pode estar relacionada com as condiçóes de higiene da granja. Pesquisas realizadas em unidades de processamento indicam que a contaminação das carcaças de frango logo após o abate normalmente é baixa e que nas etapas de escaldamento, depenagem, evisceração e embalagem é observado um aumento significativo destes microorganismos (Fuzihara et al. 2000; Corry et al. 2002).

A contaminação de frangos por Salmonella spp pode estar relacionada com a forma em que os mesmos são transportados, as aves normalmente são confinadas e aglomeradas em caixas por longas distâncias em condições inadequadas no aspecto sanitário, aumentando assim o risco de contrair infecçôes cruzadas por salmonelas. As operações de abate e processamento das carcaças também contribuem para a disseminação e multiplicação das salmonelas que podem ocorrer por meio da água de escaldagem, no processo de depenagem, na contaminação cruzada de equipamentos e utensílios contaminados, no manuseio inadequado durante o corte e evisceração e no acondicionamento que normalmente é realizado à temperatura ambiente até a sua comercialização.

Os resultados encontrados são preocupantes, pois as salmoneloses são as grandes responsáveis pela maioria das toxinfecçōes alimentares humanas e a ocorrência difundida destes microorganismos reforça a necessidade de medidas de controle.

\section{BIBLIOGRAFIA CITADA}

Almeida, I. C.; Gonçalves, P. M. R.; Franco, R. M.; Carvalho, J. C. A. P. 2000. Isolamento e identificação de Salmonella em carcaças de frango congelados e frescais, através de método rápido. Higiene Alimentar, 14(70):59-62. 
Andrews, W. H.; Hammack, T. S. 2003. Salmonella. In: Food and Drug Administration. Bacterial Analitical Manual. Revision A. 8 ed. Arlington : AOAC International. p. 501-519.

Bauemier, A. J.; Hargis, B. M.; Tsolis, R. M. 2000. Tracing the origins of Salmonella outbreaks. Science, 287: 50-52.

Capita, R.; Alonso-Calleja, C.; Garcia-Linares, M.C.; Moreno, B.; Garcia-Fernández, M.C. 2000. Salmonella y salmonelosis humana. Alimentaria, 313:91-98.

Capita, R.; Alvarez-Astorga, M.; Alonso-Calleja, C.; Moreno, B.; Garcia-Fernández, M.C. 2003. Occurrence of Salmonellae in retail carcasses and their products in Spain. . Journal of Food Microbiology, 81:169-173.

Corry, J. L. E.; Allen, V. M..; Hudson, W. R.; Breslin, M. F.; Davies, R. H. 2002. Sources of salmonella on broiler carcasses during transportation and processing: modes of contamination and methods of control. Journal of Applied Microbiology., 92:424432.

Daniels, N. A.; MacKinnon, 1.; Rowe, S. M.; Bean, N. H.; Griffin, P. M.; Mead, P. S. 2002. Foodborne disease outbreaks in United States schools. Journal of Pediatric Infectious Diseases, 21(7):623628.

dos Santos, L. R.; do Nascimento, V. P.; de Oliveira, S. D.; Rodrigues, D. P.; dos Reis, L. M.; Ribeiro, A. R.; Fernandes, S. A. 2003. Phages types of Salmonella enteritidis isolated from clinical and foods and broiler carcasses in southern Brazil. Revista do Instituto de Medicina tropical de São Paulo., 45(1):1-4.

Duitschaever, C. L. 1977. Incidence of Salmonella in retailed raw cut-up chicken. Journal of Food Protection, Ames, 40(1):191192.

FDA Center for Food Safety \& Applied Nutrition. Foodborne Pathogenic Microorganisms and Natural Toxins 1992 (Bad Bug Book).Internet [http://vm.cfsan.fda.gov/ - mow/chap1.html], 2003.

Fuzihara, T. O.; Fernandes, S. A.; Franco, B. D. 2000. Prevalence and dissemination of Salmonella serotypes along the slautering process in Brazilian small poultry slaughterhouses. Journal of Food Protection., 63(12):1749-1753.

Jay, J. M. 2000. Modern Food Microbiology. Aspen Publishers Inc. Maryland. $6^{\text {th }}$ ed. , p. 511-525.

Landgraf, M.; Franco, B. D. G. M. 1996. Doenças microbianas de origem alimentar provocadas por enteropatógenos. Revista de Ciências Farmacêuticas. São Paulo, 17(1):57-76.
Mead, P. S.; Slutsker, L.; Dietz, V.; McCaig, L. F.; Bresee, J. S..; Shapiro, C.; Griffin, P. M.; Tauxe, R. V. 1999. Food-Related Illness and Death in the United States. Emerging Infections Diseases, 5:607-625

Mikolajczyk, A.; Radkowski, M. 2002. Salmonella spp on chicken carcasses in processing plants in Poland. Journal of Food Protection, 65(9):1475-1479.

Mukesh, D. S.; Mukesh, G. S. 2002. Serotypic and antibiotic susceptibility pattern of Salmonella species isolated from cases os gastroenteritis at Infectious Disease Hospital (IDH), Delhi from 1997-2000. Journal of Communicable Diseases, 34(4):237244.

Mulder, R. W. A. W. 1999. European directives to regulate food safety. World Poult. Sci. J., 15:50-51.

Olsen, S. J.; Bishop, R.; Brenner, F. W.; Roels, T. H.; Bean, N.; Tauxe, R. V..; Slutsker, L. 2001. The Changing Epidemiology of Salmonella: Trends in Serotypes Isolates from Human in the States, 1987-1997. Journal of the Infectious Diseases, 183:753761.

Olsen, J. E.; Brown, D. J.; Madsen, M.; Bisgaard, M. 2003. Crosscontamination with Salmonella on a broiler slaughterhouse line demonstrated by use of epidemiological markets. Journal of Applied Microbiology, 95(5):826-835.

Picollo, R. C.; Pimentel, E.P.; Fávero, L.M.; Rizzo, M.A.; Pascher, D.M. 1992. Surto de salmonelose ocorrido em cantina escolar, no município de São Paulo em 1991. Higiene Alimentar., 6(23):28-30.

Tavechio, A. T.; Ghilardi, A. C.; Peresi, J. T.; Fuzihara, T. O.; Yonamine, E. K.; Jakabi, M.; Fernandes, S.A. 2002. Salmonella serotypes isolated from nonhuman sources in São Paulo, Brazil, from 1996 though 2000. Journal of Food Protection, 65(6):10411044 .

Tibaijuka, B.; Molla, B.; Hildebrandt, G.; Kleer, J. 2003. Ocurrente of Salmonellae in retail raw chicken products in Ethiopia. Berl Munch Tierarztl Wochensch, 116(1-2):55-58.

Uyttendaele, M.; De Troy, P.; Debevere, J. 1999. Incidence of Salmonella, Campylobacter jejuni, Campylobacter coli, and Listeria monocytogenes in poultry carcasses and different types of poultry products for sale on the Belgian retail market. Journal of Food Protection., 62(7):735-740.

Recebido em 22/12/2003

Aceito em 02/03/2006 